\title{
A photoacoustic-fluorescent imaging probe for proteolytic gingipains expressed by Porphyromonas gingivalis
}

\author{
Colman Moore ${ }^{[a]}$, Natalia Tjokro ${ }^{[b]}$, Brendan Zhang ${ }^{[a]}$, Mohammed Hayati ${ }^{[b]}$, Yong Cheng ${ }^{[a]}$, Kai Chiao \\ Joe Chang ${ }^{[b]}$, Casey Chen ${ }^{[b]}$, and Jesse V. Jokerst ${ }^{[\mathrm{a}],[\mathrm{c}],[\mathrm{d}] *}$
}

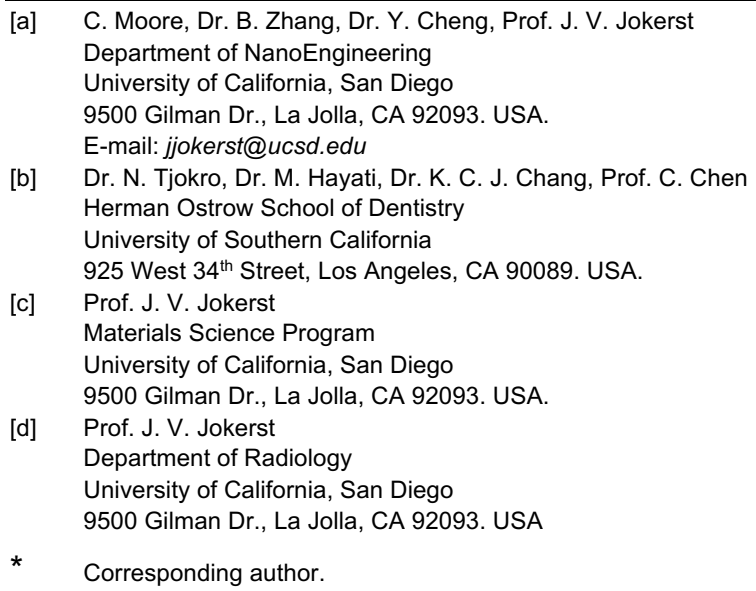

\begin{abstract}
Porphyromonas gingivalis is a keystone pathogen in periodontal disease. We herein report a dual-modal fluorescent and photoacoustic imaging probe for the detection of gingipain proteases secreted by $P$. gingivalis. This probe harnesses the intramolecular dimerization of peptide-linked cyanine dyes to induce fluorescence and photoacoustic off-states. Upon proteolytic cleavage by Argspecific gingipain (RgpB), five-fold photoacoustic enhancement and $>100$-fold fluorescence activation was measured with detection limits of $1.1 \mathrm{nM} \mathrm{RgpB}$ and $5.0 \mathrm{E} 4 \mathrm{CFU} / \mathrm{mL}$ bacteria in vitro. RgpB activity was imaged in the subgingival pocket of porcine jaws with $25 \mathrm{nM}$ sensitivity. The diagnostic efficacy of the probe was evaluated in gingival crevicular fluid (GCF) samples from subjects with $(n=14)$ and without $(n=6)$ periodontal disease, wherein activation was correlated to GPCR-based detection of $P$. gingivalis (Pearson's $r=0.71$ ). The highest activity was seen in subjects with the most severe disease. Finally, photoacoustic imaging of RgpB-cleaved probe was achieved in murine brains ex vivo, demonstrating relevance and potential utility for animal models of general infection by $P$. gingivalis, motivated by the recent biological link between gingipain and Alzheimer's disease.
\end{abstract}

\section{Introduction}

Periodontitis is a chronic inflammatory disease that affects $46 \%$ of adults in the United States and generates billions of dollars per year in direct costs ${ }^{[1]}$. The pathogenesis of the disease remains an active research topic; however, it is principally associated with a dysbiotic oral microbiome and the accompanying immune response [2]. Periodontitis-associated bacteria reside in the subgingival crevice, and their presence in biofilms and gingival crevicular fluid contribute to degradation of host tissue and deepening of the periodontal pocket [3]. When untreated, periodontitis causes oral pain, tooth loosening, and tooth loss. Furthermore, the long-term loading of the immune system has been linked to increased risks for cardiovascular disease ${ }^{[4]}$, pre-term birth ${ }^{[5]}$, cancer ${ }^{[6]}$, and even dementia ${ }^{[7]}$.

Periodontal health is measured via periodontal probing and clinical examination with metrics that include the pocket depth, clinical attachment level, bleeding on probing, tooth mobility, and inflammation. Together, these metrics are used to form a diagnosis.
In general, this established practice is functional and affordable, but pocket depth and clinical attachment level measurements suffer from relatively high inter-examiner error due to differences in probing force/angulation while also causing patient discomfort. Moreover, these techniques largely assess the effects of disease rather than using molecular diagnostics for precision health. Therefore, new techniques to detect disease at the point-of-care-particularly with utility for imaging and identification of disease at the molecular levelremain an unmet need in the field of oral health.

Many of the periodontal pathogens that have been linked to disease are anaerobic, such as Tannerella forsythia, Treponema denticola, and Porphyromonas gingivalis ${ }^{[8]}$. Among this "red complex", $P$. gingivalis is the most well-characterized: Its presence in subgingival plaque has been correlated with disease progression in longitudinal human studies ${ }^{[9]}$. As a function of their anaerobic metabolism, these pathogens secrete protease virulence factors that degrade extracellular proteins and modulate the host immune response ${ }^{[10]}$. $P$. gingivalis, in particular, is known to secrete proteases called gingipains that exhibit trypsin-like activity ${ }^{[1]}$.

Indeed, $P$. gingivalis and gingipain proteases have attracted attention both as diagnostic and therapeutic targets. A variety of naturally derived and synthetic gingipain inhibitors have been reported in the literature while demonstrating evidence for potential treatment of periodontal disease though clinical trials have been relatively rare ${ }^{[12]}$. Intriguingly, evidence of gingipains has been identified in the post-mortem brains of patients with Alzheimer's disease $(A D)$ and are the target of an ongoing $A D$ clinical trial for a small molecule gingipain inhibitor ${ }^{[13]}$. A parallel research effort is targeting $P$. gingivalis directly with an antibody therapy ${ }^{[14]}$.

From a diagnostic perspective, advances in gingipain detection have included the development of substrates and paper-based assays for in situ analysis ${ }^{[15]}$, a plasmonic nanosensor ${ }^{[16]}$, and a gingipain-responsive/drug-loaded hydrogel ${ }^{[17]}$. The goal of this study was to develop an activatable probe for gingipains with utility for in vivo imaging-such work was motivated by its potential as a clinical tool for periodontal diagnosis and as a research tool for investigation of the role of gingipains in periodontitis and other diseases.

Photoacoustic imaging is particularly attractive because it augments the existing strengths of ultrasound-good tissue 


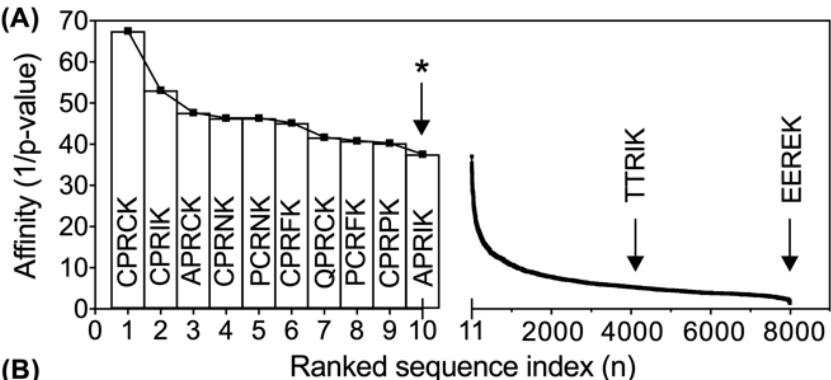

(B)

\begin{tabular}{|c|c|c|}
\hline Peptide & $\begin{array}{c}\text { Net } \\
\text { charge }\end{array}$ & $\begin{array}{c}\text { RgpB affinity (p- } \\
\text { value) }\end{array}$ \\
\hline APR/IK & +2 & 0.02658 \\
\hline $\mathrm{TTR} / \mathrm{IK}$ & +2 & 0.1866 \\
\hline $\mathrm{EER} / \mathrm{EK}$ & -1 & 0.6872 \\
\hline
\end{tabular}

(C)

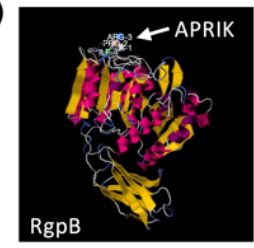

(D) C2A Probe

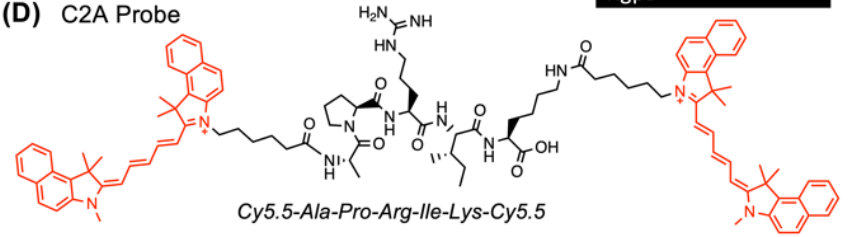

(E)

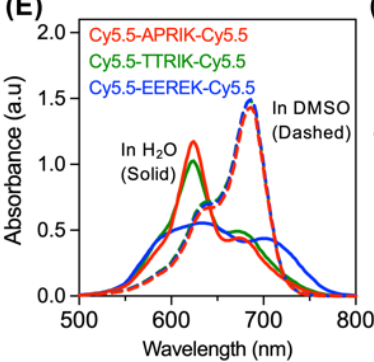

(F)

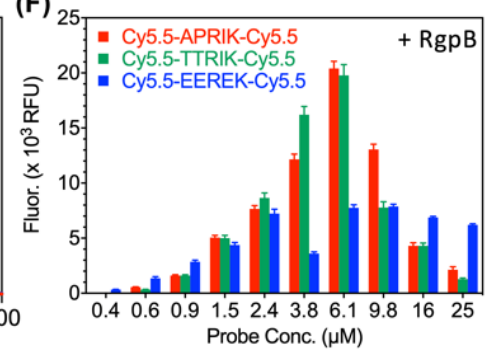

Figure 1. Structural selection and optical validation of photoacoustic/fluorescent peptides for RgpB. (A) Rankings of modeled peptide affinities for RgpB using an open-source structural model. All 8,000 five-residue peptides with a $\mathrm{P} 1$-arginine and $\mathrm{C}$-terminal lysine were screened against RgpB (PDB: 1cvr). The affinities were plotted as the inverse $p$-values corresponding to the statistical significance of the peptideprotein interaction. APRIK was identified as the top candidate containing no cysteine residues (cysteine-containing sequences were excluded due to their potential for disulfide bridging). (B) APRIK, TTRIK (median affinity), and EEREK (lowest affinity) were selected for double conjugation with Cy5.5. (C) Structural model of top match (APRIK) with RgpB indicates preferential affinity for the catalytic domain. (D) Molecular structure of the dye-conjugated peptide, [Cy5.5]2[APRIK]. (E) Absorbance spectra of the conjugates in DMSO vs. $\mathrm{H}_{2} \mathrm{O}$ (1\% DMSO) at equimolar concentrations (15 $\mu \mathrm{M})$. (F) Fluorescence intensities for each conjugate after $1.5 \mathrm{~h}$ at a range of concentrations incubated with $25 \mathrm{nM}$ RgpB.

penetration, low cost, and real-time monitoring. It can use both exogenous and endogenous contrast based on optical absorption. Many small molecule and nanoparticle contrast agents have been engineered for photoacoustic imaging and activatable probes for molecular imaging are particularly desirable ${ }^{[18]}$. Further, the applications of acoustic imaging and nanoscale materials have been expanding ${ }^{[19]}$ but they have not yet been combined for oral imaging. In previous work, we introduced a dye-peptide scaffold that exploits the intramolecular coupling of cyanine dyes to generate photoacoustic and fluorescent signal upon proteolysis by trypsin. Here, we leveraged this approach to create an activatable photoacoustic and fluorescent molecular imaging agent for gingipain proteases.
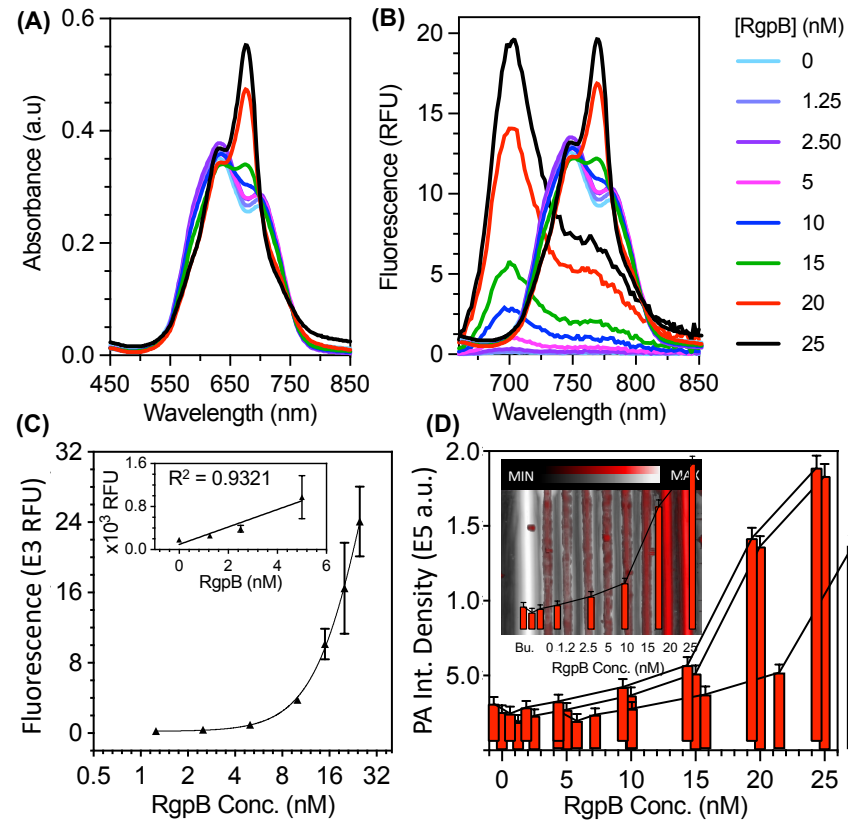

Figure 2. Optical and photoacoustic limits of detection for C2A with recombinant gingipain (RgpB). (A) Absorbance spectra of $\mathrm{C} 2 \mathrm{~A}$ incubated with $0-25 \mathrm{nM} \mathrm{RgpB}$ after 90 minutes. (B) Fluorescence spectra (ex: $600 \mathrm{~nm}$, em: $700 \mathrm{~nm}$ ) of the samples after 90 minutes. (C) Fluorescence limit of detection for RgpB via fluorescence area scan (ex: 625, em: $700 \mathrm{~nm}, \mathrm{LOD}=1.1 \mathrm{nM}$, linear range: $0-5 \mathrm{nM}, \mathrm{R}^{2}=0.93, \mathrm{n}=2$, error $=\mathrm{SEM})$. (D) Photoacoustic-ultrasound image with quantitated intensities for RgpB from $0-25 \mathrm{nM}$ (ex: $680 \mathrm{~nm}$, LOD = $15 \mathrm{nM}$, inset = raw photoacoustic ultrasound image, $\mathrm{Bu} .=$ buffer $)$.

To select a gingipain-cleavable peptide substrate, we first applied a structural model of peptide-protein affinity to screen a series of pentapeptides for their affinity to the Arg-specific cysteine protease gingipain $\mathrm{R}$ (RgpB, PDB: 1CVR) ${ }^{[20]}$. The RgpB protease is composed of a 435-residue, single-chain polypeptide that forms a catalytic domain and an immunoglobulin-like domain ${ }^{[21]}$. The peptide candidates were generated with three constraints: a five-residue length, an arginine at the third residue $\left(P_{1}\right)$, and a lysine at the fifth residue (C-terminus, $\mathrm{P}_{2}$ '). The peptide length was restricted to facilitate intramolecular interaction between $\mathrm{N}$ and $\mathrm{C}$ terminal dyes while reducing the likelihood of cleavage by off-target proteases. The central arginine was necessary for cleavage by $\mathrm{RgpB}$, and the Cterminal lysine was chosen for its reactive free amine. These conditions allowed us to generate 8,000 possible sequences that were screened for affinity to RgpB using an open-source structural model (PepSite 2.0) based upon a library of known peptide-protein complexes ${ }^{[20 b]}$. The results were plotted as the inverse $p$-value to signify relative affinity (Fig. 1A) where the $p$-value represents the statistical significance for the overall score of a given binding site defined by Petsalaki et al. ${ }^{[22]}$. Of these peptides, the top result that did not contain a cysteine (excluded to reduce effects from dithiol coupling) was APRIK ( $p$-value 0.0266) and was selected for probe synthesis. Additionally, the median result (TTRIK ( $p$-value: 0.1866)) and last result (EEREK ( $p$-value: 0.6872$)$ ) were synthesized and 
(A)

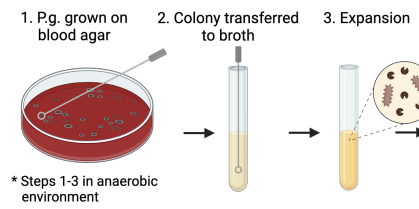

(B)

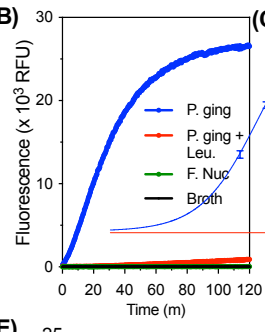

(E)
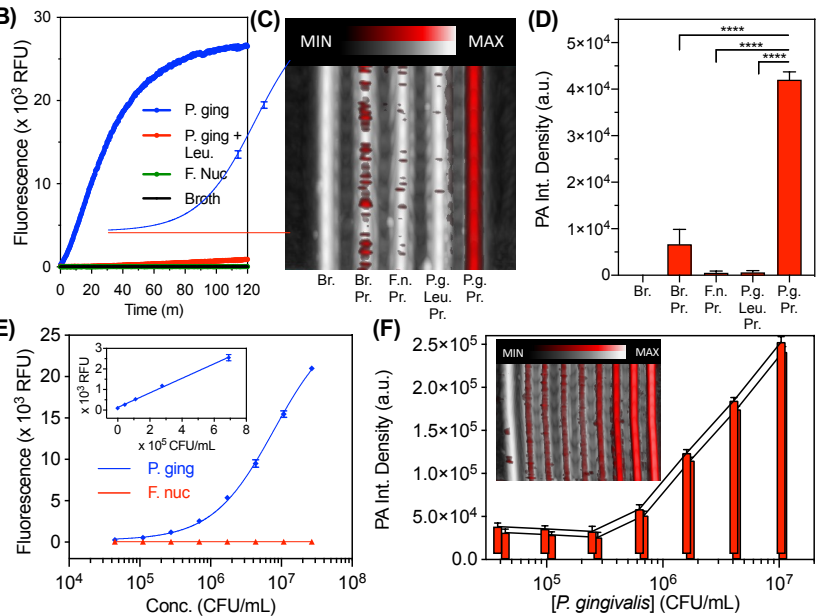

Figure 3. Optical and photoacoustic properties of C2A with bacterial supernatants from cultured $\boldsymbol{P}$. gingivalis. (A) Schematic of workflow for bacterial growth and assay. The same steps were performed for $P$. gingivalis and $F$. nucleatum. (B) Kinetics of C2A $(10 \mu \mathrm{M})$ fluorescence activation with $P$. gingivalis supernatant, $P$. gingivalis supernatant with inhibitor (50 $\mathrm{\mu M}$ leupeptin), F. nucleatum supernatant, and culture broth alone (tryptic soy broth). Supernatants were assayed at $37^{\circ} \mathrm{C}$ after $50 \%$ $(\mathrm{v} / \mathrm{v}$ ) dilution in buffer (final conditions: $2.5 \mathrm{E} 7 \mathrm{CFU} / \mathrm{mL}$ bacteria, $20 \mathrm{mM}$ Tris, $1 \mathrm{mM}$ DTT, 2\% DMSO, pH 7.3) (C) Photoacoustic-ultrasound MIP image of the samples with 680-nm pulsed excitation. $\mathrm{Br}=\mathrm{Broth}, \mathrm{Pr}=$ Probe, $\mathrm{F} . \mathrm{n}$. = F. nucleatum, P. g. = P. gingivalis, Leu = Leupeptin. (D) Quantitation of Panel $D$ via integrated pixel density. Error bars $=S D$, asterisks denote significant difference (unpaired two-tailed t-test, p-value $<0.001$ ). (E) Fluorescence limit of detection for $P$. gingivalis supernatants $(\mathrm{LOD}=4.4 \mathrm{E} 4$ $\mathrm{CFU} / \mathrm{mL}$, linear range: $\left.0-6.9 \mathrm{E} 5 \mathrm{CFU} / \mathrm{mL}, \mathrm{R}^{2}=0.99\right)$. (F) PA image and quantitation of the same samples (ex: $680 \mathrm{~nm}$ ) via integrated density (LOD = 4.1E5 CFU $/ \mathrm{mL}$, linear range: 0 - 4.3E6 CFU $/ \mathrm{mL}, \mathrm{R}^{2}=0.94$ ).

conjugated with dyes to serve as experimental controls for the model predictions (Fig. 1B). Visualization of the APRIK-RgpB interaction demonstrated that the peptide was predicted to bind the catalytic domain of RgpB (Fig. 1C). The three candidate peptides were used to synthesize homodimer probes [Cy5.5] 2 [APRIK], [Cy5.5] 2 [TTRIK], and $[\mathrm{Cy} 5.5]_{2}[\mathrm{EEREK}]$, referred throughout as $\mathrm{C} 2 \mathrm{~A}, \mathrm{C} 2 \mathrm{~T}$, and $\mathrm{C} 2 \mathrm{E}$, respectively (Fig. S1). RP-HPLC retention times for the conjugates decreased slightly from C2A (11.8 $\mathrm{min})$ to $\mathrm{C} 2 \mathrm{~T}$ (11.7 $\mathrm{min})$ to C2E (10.9 $\mathrm{min}$ ), corresponding to the increasing hydrophilicity of the residues in each peptide (Fig. S2A-C); the structures of the probes were confirmed with ESI-MS (Fig. S2D-F).

The absorbance maxima of the conjugates in water were blueshifted relative to their spectra in DMSO (Fig. 1E) - a solvatochromic effect indicative of aromatic dye stacking ${ }^{[23]}$. This blue shift confirmed intramolecular dye coupling, i.e., DMSO promotes intramolecular separation of the dyes by neutralizing their attractive $\pi-\pi$ interactions, thus mimicking the effect of proteolytic cleavage of the peptide linker. Indeed, the fluorescence of the intact conjugates was also selfquenched but was activated upon incubation with RgpB: We measured the fluorescence from each conjugate at a range of concentrations with constant RgpB and observed stronger foldenhancement for $\mathrm{C} 2 \mathrm{~A} / \mathrm{C} 2 \mathrm{~T}$ than $\mathrm{C} 2 \mathrm{E}$ (Fig. 1F). While $\mathrm{C} 2 \mathrm{~A}$ and $\mathrm{C} 2 \mathrm{~T}$ performed similarly in this comparison, we selected C2A for further
(A)

(B)

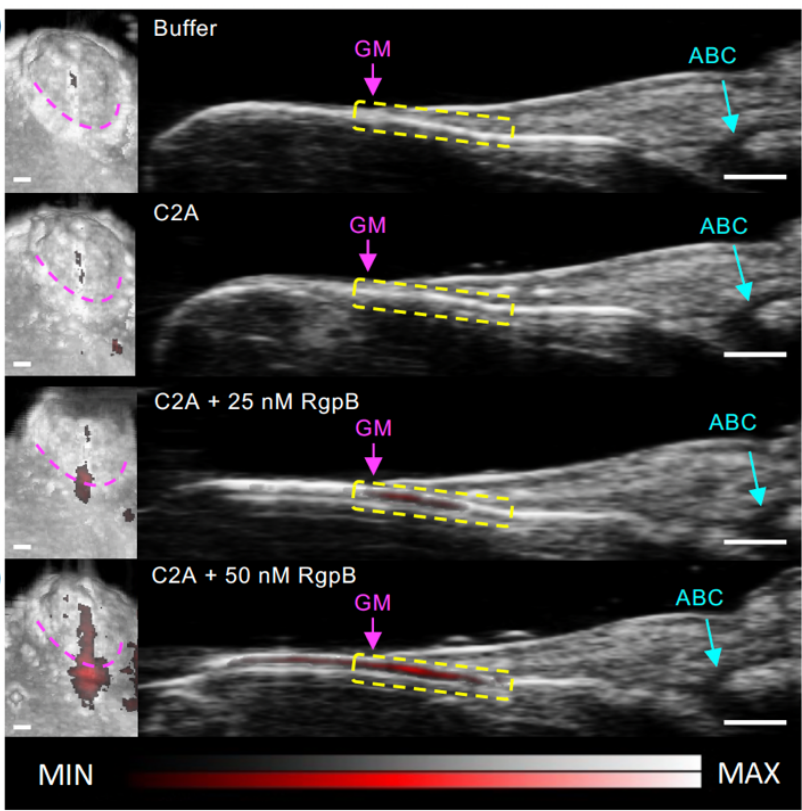

(E)

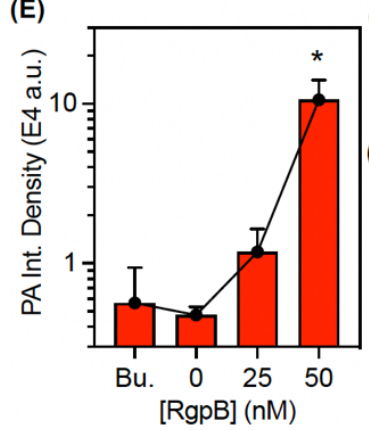

(F)

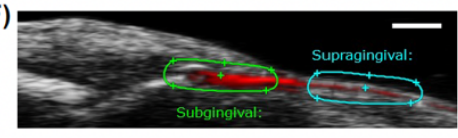

(G)

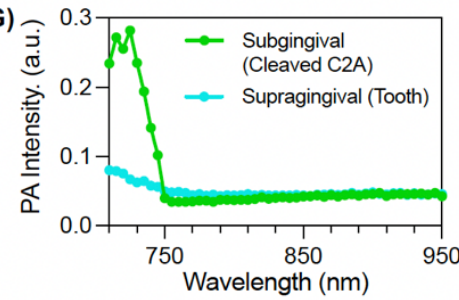

Figure 4. Photoacoustic-ultrasound imaging of RgpB-activated C2A probe in the gingival sulci of porcine jaws. (A) Left: 3D rendering of a mandibular second molar with PA signal (red) overlay on the US image (grayscale) following administration of Tris buffer at the gingival margin (pink). Right: cross-sectional image of the midsagittal plane of the tooth with the gingival margin (GM), alveolar bone crest (ABC), and gingival sulcus (yellow) labeled. (B-D) Images of the same site following administration of (B) C2A alone, (C) C2A + $25 \mathrm{nM} \mathrm{RgpB}$, and (D) C2A + $50 \mathrm{nM} \mathrm{RgpB}$. The sulcus was irrigated with water between imaging events. (E) Quantitation of the PA signal via integrated density for the maximum intensity projection of each PA image ( $n=3$ mandibles). (F) PA-US spectral image of subgingival signal corresponding to injected $\mathrm{C} 2 \mathrm{~A}$ (green $\mathrm{ROI}$ ) and supragingival signal corresponding to background from the tooth surface (teal). (G) PA spectra of the regions in Panel F. Scale bars $=1 \mathrm{~mm}$.

development due to its higher predicted affinity for RgpB and higher signal to background ratio at concentrations $>6 \mu \mathrm{M}$. The decreased activation at higher concentrations was caused by increased selfquenching of the probes, though this is dependent upon the amount of DMSO in the mixtures. Upon incubation of C2A with RgpB, the absorbance maxima of the dyes at $680 \mathrm{~nm}$ were recovered with increasing concentrations of protease (Fig. 2A). The fluorescence emission at $700 \mathrm{~nm}$ was also proportionally enhanced (Fig. 2B). The fluorescence limit of detection was $1.1 \mathrm{nM}$ (linearity $0-5 \mathrm{nM}$ ) (Fig. 2C). Additionally, the photoacoustic intensity of the samples excited at $680 \mathrm{~nm}$ was proportional to their absorption (Fig. 2A, D), and the photoacoustic limit of detection was $10 \mathrm{nM}$ RgpB.

To further verify the probe's sensitivity and selectivity for gingipains associated with $P$. gingivalis, we grew and isolated bacterial supernatants from both $P$. gingivalis and another oral anaerobe, F. nucleatum (Fig. 3A). F. nucleatum is a good negative control because it is also commonly identified in the gingival sulcus 

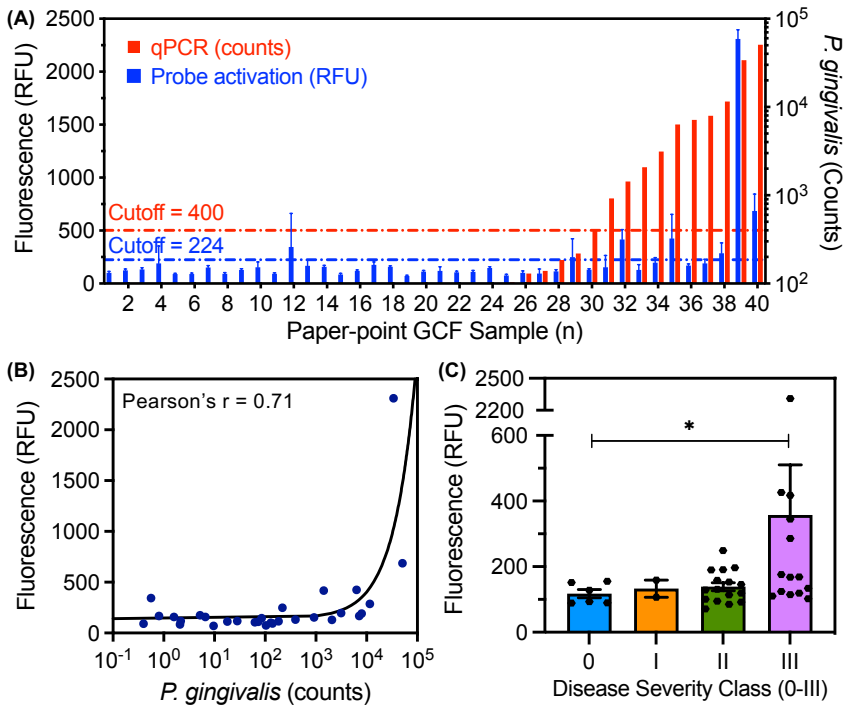

Figure. 5. Comparison between qPCR for $P$. gingivalis and fluorescence of C2A probe in GCF samples from a set of 20 subjects ( $n=\mathbf{4 0}$ tooth sites). (A) Number of $P$. gingivalis cells (red) and probe activation (blue, gingipain activity) for each GCF sample (error bars $=$ SD of fluorescence area scans, $n=13$ points per well). (B) Correlation between number of $P$. gingivalis cells and probe activation (Pearson's $r=0.71$, twotailed, $\mathrm{p}<0.0001$ ). (C) Probe activation for individual tooth sites from human subjects as a function of clinically diagnosed periodontal disease severity (Welch's one-tailed t-test, $90 \%$ confidence: $p$-value $=0.07<0.10$, error bars $=$ SEM).

but is a saccharolytic and commensal bacterium known to not secrete gingipains ${ }^{24]}$. These anaerobes were first grown on blood agar and enumerated from liquid suspensions via optical density after development of standard curves (Fig. S3, Fig. S4). The presence of Arg-specific gingipain in the $P$. gingivalis cultures was confirmed with a commercially available enzyme-linked immunoassay (ELISA) kit (Fig. S5); in addition, activity was measured by incubation with a commercially available fluorescent substrate, Boc-Phe-Ser-Arg-AMC, as previously described ${ }^{[25]}$ (Fig. S6). Then, upon incubation of the C2A probe with $P$. gingivalis supernatant, we directly observed cleavage of intact C2A $\left(T_{R}=21.2 \mathrm{~min}\right)$ into Cy5.5-APR $\left(T_{R}=17.2 \mathrm{~min}\right.$, $\left.[\mathrm{M}+2 \mathrm{H}]^{2+}=412.91 \mathrm{~m} / \mathrm{z}\right)$ and IK-Cy5.5 $\left(\mathrm{T}_{\mathrm{R}}=18.2 \mathrm{~min},[\mathrm{M}+2 \mathrm{H}]^{2+}=\right.$ $454.93 \mathrm{~m} / \mathrm{z}$ ) fragments with HPLC and ESI-MS (Fig. S7), thus demonstrating the expected activity of Arg-gingipain in the bacterial supernatant and intended cleavage of C2A. Indeed, the probe activated fluorescence 135-fold over the course of 2 hours, corresponding to enhanced emission at $700 \mathrm{~nm}$ and absorbance at $680 \mathrm{~nm}$ (Fig. 3B, Fig. S8); this activation was reduced by $97 \%$ upon coincubation with leupeptin-a known gingipain inhibitor ${ }^{[26]}$ (Fig. 3B). The fluorescence was not activated by $F$. nucleatum. As with fluorescence, we observed an increasing trend for the photoacoustic intensities of the samples excited at $680 \mathrm{~nm}$, thus demonstrating selective photoacoustic imaging of gingipains from $P$. gingivalis (Fig. 3C-D). The limits of detection for the bacteria were tested via serial dilution of the supernatants in broth and determined to be $4.4 \mathrm{E} 4$ $\mathrm{CFU} / \mathrm{mL}$ via fluorescence and 4.1E5 CFU/mL via photoacoustics (Fig. 3E-F, Fig. S9).

To date, reported strategies for measurement of gingipain activity have used in vitro detection methods, including a nanobody immunoassay ${ }^{[15 a]}$, an electrochemical biosensor ${ }^{[27]}$, fluorogenic dipeptides $^{[15 b]}$, peptide-functionalized magnetic nanobeads ${ }^{[28]}$, and refractometry of protein-functionalized gold nanoparticles ${ }^{[16]}$. These have reported detection limits of $7.81 \mathrm{E} 6 \mathrm{CFU} / \mathrm{mL}$ bacteria, $5 \mathrm{E} 5$

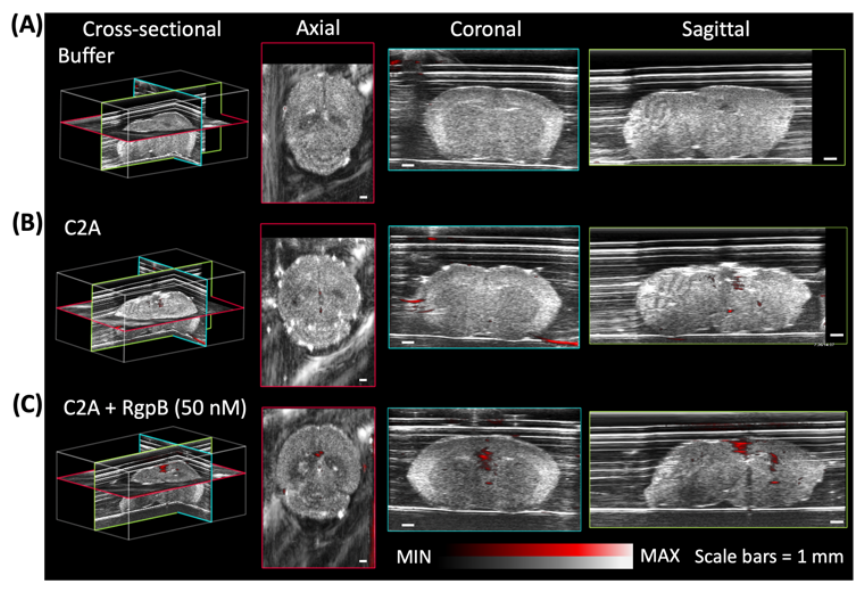

Figure 6. Photoacoustic imaging of RgpB-activated C2A probe in murine brains. Photoacoustic-ultrasound images of fixed murine brains injected at the lambda point with (A) buffer, (B) C2A probe, and (C) C2A probe + RgpB, show enhanced intraparenchymal photoacoustic signal for RgpB-cleaved probe. Photoacoustic intensity (red) is overlaid on ultrasound intensity (grayscale). Scale bars $=1 \mathrm{~mm}$.

$\mathrm{CFU} / \mathrm{mL}$ bacteria, $1 \mathrm{E} 5 \mathrm{CFU} / \mathrm{mL}$ bacteria, $49 \mathrm{CFU} / \mathrm{mL}$ bacteria, and $4.3 \mathrm{nM} \mathrm{Kgp} \mathrm{(CFU/mL} \mathrm{not} \mathrm{reported),} \mathrm{respectively.} \mathrm{While} \mathrm{the} \mathrm{C2A} \mathrm{probe}$ has comparable sensitivity to these in vitro sensors (fluorescence LOD: 4.4E4 CFU/mL and 1.1 nM RgpB, photoacoustic LOD: 4.1E5 $\mathrm{CFU} / \mathrm{mL}$ and $15 \mathrm{nM} \mathrm{RgpB}$ ), it is the first reported gingipain probe suitable for photoacoustic imaging while also achieving a dual-modal fluorescence readout, with applicability for in vivo oral photoacoustic imaging, a technique that is gaining preclinical traction ${ }^{[29]}$. The added value of imaging is the monitoring of disease progression or response to therapy with the spatial integration of anatomic markers of disease. Indeed, to characterize the imaging performance of the C2A probe in relevant oral anatomy, it was used to resolve the periodontal pocket/gingival sulcus of intact porcine jaws with photoacousticultrasound imaging (Fig. 4). Here, buffer, C2A, and C2A + RgpB (25 and $50 \mathrm{nM}$ ), were irrigated sequentially into the gingival sulcus of the second molar of a porcine mandible $(n=3)$. 3D photoacousticultrasonographs of the tooth/gingiva were generated (Fig. 4A, left) and anatomical markers were readily resolved in the midsagittal cross sections (Fig. 4A, right), including the gingival margin ( $G M$, pink) and alveolar bone crest (ABC, teal). The uncleaved $C 2 A$ probe did not possess significantly more photoacoustic signal (red) than buffer alone (Fig. 4B). However, C2A activated with 25-50 nM RgpB generated clear and increasing subgingival photoacoustic signal (Fig. 4C-E, yellow boxes), representing the subgingival distribution of RgpB-cleaved probe. In addition, spectral imaging could distinguish the imaging signal from cleaved $\mathrm{C} 2 \mathrm{~A}(<750 \mathrm{~nm})$ from the relatively flat spectra from supragingival signal caused by tooth staining (Fig. 4F-G). Overall, this experiment demonstrates the ability to image the spatial distribution of subgingival gingipain activity in relation to key landmarks of oral anatomy while achieving low nanomolar sensitivity.

In a study by Guentsch et al., ELISA was used to identify micromolar concentrations of gingipain in gingival crevicular fluid (GCF) collected with paper point sampling from patients with periodontal disease ${ }^{[30]}$. This is well above the low nanomolar detection limits of C2A for RgpB: Therefore, to evaluate the diagnostic efficacy of the $\mathrm{C} 2 \mathrm{~A}$ probe in clinically relevant samples, we collected GCF from 40 tooth sites in a set of 20 subjects, comprising both healthy patients and individuals with symptoms of periodontal disease sampled at a dental clinic. The GCF samples were assayed with both qPCR and C2A via fluorescence to measure the number of $P$. 
gingivalis cells and proteolytic gingipain activity, respectively. Of these, $25 \%(10 / 40)$ contained $P$. gingivalis via $\mathrm{qPCR}$ and these were considered positives (Fig. 5A). Gingipain activity via C2A fluorescence was correlated with the PCR results (Pearson's $r=0.71$, Fig. 5B), albeit with lower sensitivity: Fluorescence activation was observed in $5 / 10$ of the positives and $2 / 30$ of the negatives, corresponding to a detection rate of $50 \%$ and a false positive rate of $6.67 \%$ (Fig. 5A). However, the higher sensitivity of qPCR was expected given its inherent signal amplification mechanism. Another difference is that while GPCR may reflect the amount of live and dead cells, it is not a measurement of the active gingipain activity that is evidenced to play a direct role in the pathogenic process of periodontal disease ${ }^{[31]}$. The activity data was also analyzed with respect to disease severity for each tooth site (Fig. 5C). Interestingly, gingipain activity was primarily observed in the GCF from Class III sites (with the greatest total facial CAL). Though half of these sites did not exhibit gingipain activity, these results support the hypothesis that local gingipain activity may contribute to more severe periodontal damage.

Lastly, the potential role of $P$. gingivalis and gingipains in neurological pathologies, especially Alzheimer's disease, is of mounting research interest ${ }^{[13,32]}$. Photoacoustic imaging is well-suited for real-time imaging and monitoring of murine disease models, and thus we performed proof of concept imaging of cleaved and uncleaved probe in extracted murine brains (fixed in 1\% agar). The C2A probe was first incubated with $\mathrm{RgpB}$ at increasing probe concentrations to confirm cleavage at sufficient concentrations for imaging in animal tissue (Fig. S10A-B), and the highest tested concentration (30 $\mu \mathrm{M})$ was chosen for injection (Fig. S10C). Subsequently, aliquots of buffer, $\mathrm{C} 2 \mathrm{~A}$, and $\mathrm{C} 2 \mathrm{~A}+\mathrm{RgpB}$ (pre-incubated and monitored for $2 \mathrm{~h}$ ) were injected into the lambda points of respective brains (Fig. S10D)these were then imaged in 3D with a photoacoustic-ultrasound scanner at $680 \mathrm{~nm}$ using sonography gel for acoustic coupling. Negligible photoacoustic signal was detected in the buffer-injected brain (Fig. 6A), while minor background was observed for the uncleaved probe (Fig. 6B). The strongest signal was detected from the brain injected with $\mathrm{C} 2 \mathrm{~A}+\mathrm{RgpB}$, visible in axial, coronal, and sagittal cross-sections of the tissue (Fig. 6C). Further, spectral photoacoustic imaging of the injected brains allowed signal from C2A to be distinguished from background by its characteristic absorption/photoacoustic peak in the near infrared (Fig. S11). These experiments demonstrate that the $\mathrm{C} 2 \mathrm{~A}$ probe could have value as a research tool for gingipain imaging in more complex models of infection for Alzheimer's disease pathogenesis. Future studies may integrate the probe with in vivo models of $P$. gingivalis infection, though potential limitations include issues that affect many smallmolecule photoacoustic probes, including low signal to background ratio in blood at low concentrations and photoinstability associated with the dissociation of conjugated $\pi$ electrons following absorption [23,33]. Nevertheless, proof-of-concept imaging utility was demonstrated in the oral cavity and brain parenchyma using resected porcine jaws and murine brains, respectively. Lastly, in future efforts to improve sensitivity to $P$. gingivalis, a lysine residue could be included in the peptide linker for cleavage by Lys-gingipain (Kgp), in addition to $\mathrm{D}$-amino acids for increased bacterial specificity ${ }^{[34]}$.

In summary, a molecular imaging probe, C2A, was designed and synthesized to harness the intramolecular dimerization of peptide-linked cyanine dyes to induce fluorescence and photoacoustic off-states. Upon proteolytic cleavage by Arg-specific gingipain (RgpB), 5-fold photoacoustic enhancement and $>100$-fold fluorescence enhancement was achieved with detection limits of 1.1
$\mathrm{nM} \mathrm{RgpB}$ and 4.4E4 CFU/mL bacteria. RgpB activity was imaged in the subgingival pocket of porcine mandibles with $25 \mathrm{nM}$ sensitivity. The diagnostic efficacy of the probe was evaluated in gingival crevicular fluid (GCF) samples from subjects with $(n=14)$ and without $(n=6)$ periodontal disease; activation correlated to qPCR-based detection of $P$. gingivalis (Pearson's $r=0.71$ ), and activity was highest in subjects with the most severe disease progression. Lastly, photoacoustic imaging of RgpB-cleaved probe was demonstrated in murine brains ex vivo, thus demonstrating future utility for imaging studies of general infection by $P$. gingivalis.

\section{Conflicts of interest}

Jesse V. Jokerst is a co-founder of StyloSonic, LLC.

\section{Acknowledgements}

The authors acknowledge funding from the National Institutes of Health via R21 DE029917, R21 DE029025, UL1TR001442, DP2 HL137187-S1, and S10 OD021821. The content is solely the responsibility of the authors and does not necessarily represent the official views of the NIH. This work was supported in part by the National Science Foundation Graduate Research Fellowship Program under grant no. DGE-1650112. C.M. graciously acknowledges funding from the ARCS (Achievement Reward for College Scientists) Foundation. J.V.J. acknowledges the generous support of The Shiley Foundation.

Keywords: Biosensors - Fluorescent probes - Gingipain • Imaging agents $\bullet$ Photoacoustic imaging

\section{Entry for Table of Contents}

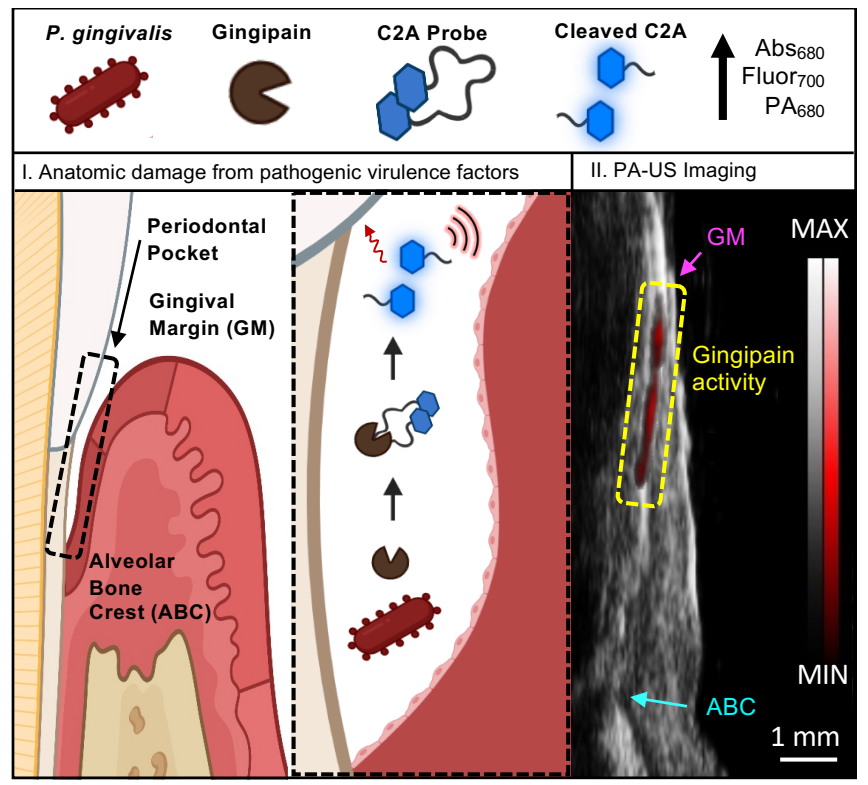




\section{References}

[1] a) P. I. Eke, B. A. Dye, L. Wei, G. D. Slade, G. O Thornton-Evans, W. S. Borgnakke, G. W. Taylor, R. C. Page, J. D. Beck, R. J. Genco, J. Periodontol. 2015, 86, 611-622; b) S. Listl, J. Galloway, P. Mossey, W. Marcenes, J. Dent. Res. 2015, 94, 1355-1361.

[2] G. Hajishengallis, Nat. Rev. Immuno. 2014, 15, 30.

[3] M. Kuboniwa, R. J. Lamont, Periodontol. 2000 2010, 52, 38-52.

[4] W.-D. Leng, X.-T. Zeng, J. S. Kwong, X.-P. Hua, Int. J. Cardiol. 2015, 201, 469-472.

[5] M. A. Nazir, Int. J. Health Sci. 2017, 11, 72-80.

[6] D. Michaud, K. Kelsey, E. Papathanasiou, C. Genco, E. Giovannucci, Ann. Oncol. 2016, 27, 941-947.

[7] Y. T. Lee, H. C. Lee, C. J. Hu, L. K. Huang, S. P. Chao, C. P. Lin, E. C. Y. Su, Y. C. Lee, C. C. Chen, J. Am. Geriatr. Soc. 2017, 65, 301-305.

[8] G. Hajishengallis, R. J. Lamont, Mol. Oral Microbiol. 2012, 27, 409-419.

[9] a) S. J. Byrne, S. G. Dashper, I. B. Darby, G. G. Adams, B. Hoffmann, E. C. Reynolds, Oral Microbiology and Immunology 2009, 24, 469-477; b) J. S. Kinney, T. Morelli, M. Oh, T. M. Braun, C. A. Ramseier, J. V. Sugai, W. V. Giannobile, J. Clin. Periodontol. 2014, 41, 113-120.

[10] D. T. Graves, Y. Jiang, C. Genco, Curr. Opin. Infect. Dis. 2000, 13, 227-232.

[11] T. Imamura, J. Periodontol. 2003, 74, 111-118.

[12] a) I. Olsen, J. Potempa, J. Oral Microbiol. 2014, 6, 24800; b) S. Kataoka, A. Baba, Y. Suda, R. Takii, M. Hashimoto, T. Kawakubo, T. Asao, T. Kadowaki, K. Yamamoto, The FASEB Journal 2014, 28, 3564-3578; c) T. Kadowaki, A. Baba, N. Abe, R. Takii, M. Hashimoto, T. Tsukuba, S. Okazaki, Y. Suda, T. Asao, K. Yamamoto, Mol. Pharmacol. 2004, 66, 1599.

[13] S. S. Dominy, C. Lynch, F. Ermini, M. Benedyk, A. Marczyk, A. Konradi, M. Nguyen, U. Haditsch, D. Raha, C. Griffin, L. J. Holsinger, S. Arastu-Kapur, S. Kaba, A. Lee, M. I. Ryder, B. Potempa, P. Mydel, A. Hellvard, K. Adamowicz, H. Hasturk, G. D. Walker, E. C. Reynolds, R. L. M. Faull, M. A. Curtis, M. Dragunow, J. Potempa, Science Advances 2019, 5, eaau3333.

[14] P. L. Nara, D. Sindelar, M. S. Penn, J. Potempa, W. S. T. Griffin, Journal of Alzheimer's Disease 2021, 82, 14171450.

[15] a) S. Alhogail, G. A. Suaifan, S. Bizzarro, W. E. Kaman, F. J. Bikker, K. Weber, D. Cialla-May, J. Popp, M. Zourob, Microchimica Acta 2018, 185, 149; b) W. E. Kaman, F. Galassi, J. J. de Soet, S. Bizzarro, B. G. Loos, E. C. Veerman, A. van Belkum, J. P. Hays, F. J. Bikker, J. Clin. Microbiol. 2011, JCM. 05313-05311.

[16] A. Svärd, J. Neilands, E. Palm, G. Svensäter, T. Bengtsson, D. Aili, ACS Applied Nano Materials 2020, 3, 9822-9830.

[17] S. Liu, Y.-n. Wang, B. Ma, J. Shao, H. Liu, S. Ge, ACS Applied Materials \& Interfaces 2021, 13, 36880-36893. Q. Miao, K. Pu, Bioconjugate Chemistry 2016, 27, 28082823.

[19] a) R. N. AlKahtani, The Saudi Dental Journal 2018, 30 107-116; b) M. Hannig, C. Hannig, Nature Nanotechnology 2010, 5, 565-569; c) H.-L. A. Chan, O. D. Kripfgans.

[20] a) B. P. Joshi, J. Zhou, A. Pant, X. Duan, Q. Zhou, R. Kuick, S. R. Owens, H. Appelman, T. D. Wang, Bioconjugate Chemistry 2016, 27, 481-494; b) L. G. Trabuco, S. Lise, E. Petsalaki, R. B. Russell, Nucleic Acids Res. 2012, 40, W423-W427.

[21] A. Eichinger, H.-G. Beisel, U. Jacob, R. Huber, F.-J. Medrano, A. Banbula, J. Potempa, J. Travis, W. Bode, The EMBO Journal 1999, 18, 5453-5462.

[22] E. Petsalaki, A. Stark, E. García-Urdiales, R. B. Russell, PLOS Computational Biology 2009, 5, e1000335.

[23] C. Moore, R. M. Borum, Y. Mantri, M. Xu, P. Fajtová, A. J. O'Donoghue, J. V. Jokerst, ACS Sens. 2021, 6, 23562365.

[24] Y.-J. Jung, H.-K. Jun, B.-K. Choi, J. Oral Microbiol. 2017, 9, 1320193. 2014, 14, 1-9.

[26] M.-A. Houle, D. Grenier, P. Plamondon, K. Nakayama, FEMS Microbiol. Lett. 2003, 221, 181-185.

[27] S. Park, K. Park, H. S. Na, J. Chung, H. Yang, Anal. Chem. 2021, 93, 5644-5650.

[28] P. D. Skottrup, P. Leonard, J. Z. Kaczmarek, F. Veillard, J. J. Enghild, R. O'Kennedy, A. Sroka, R. P. Clausen, J. Potempa, E. Riise, Anal. Biochem. 2011, 415, 158-167.

[29] a) C. Moore, Y. Bai, A. Hariri, J. B. Sanchez, C.-Y. Lin, S. Koka, P. Sedghizadeh, C. Chen, J. V. Jokerst, Photoacoustics 2018, 12, 67-74; b) M. Mozaffarzadeh, C. Moore, E. B. Golmoghani, Y. Mantri, A. Hariri, A. Jorns, L. Fu, M. D. Verweij, M. Orooji, N. de Jong, J. V. Jokerst, Biomedical Optics Express 2021, 12, 1543-1558; c) C. Lin, F. Chen, A. Hariri, C. Chen, P. Wilder-Smith, T. Takesh, J. Jokerst, J. Dent. Res. 2018, 97, 23-30.

[30] A. Guentsch, M. Kramesberger, A. Sroka, W. Pfister, J. Potempa, S. Eick, J. Periodontol. 2011, 82, 1051-1060.

[31] D. Grenier, V. Dang La, Curr. Drug Targets 2011, 12, 322331.

[32] a) H. Nakanishi, S. Nonaka, Z. Wu, CNS \& Neurological Disorders-Drug Targets (Formerly Current Drug TargetsCNS \& Neurological Disorders) 2020, 19, 495-502; b) M. I. Ryder, J. Periodontol. 2020, 91, S45-S49; c) U. Haditsch, T. Roth, L. Rodriguez, S. Hancock, T. Cecere, M. Nguyen, S. Arastu-Kapur, S. Broce, D. Raha, C. C. Lynch, Journal of Alzheimer's Disease 2020, 75, 1361-1376.

[33] T. Stahl, T. Allen, P. Beard, in Photons Plus Ultrasound: Imaging and Sensing 2014, Vol. 8943, International Society for Optics and Photonics, 2014, p. 89435H.

[34] E. Kaman Wendy, F. Galassi, J. de Soet Johannes, S. Bizzarro, G. Loos Bruno, C. I. Veerman Enno, A. van Belkum, P. Hays John, J. Bikker Floris, J. Clin. Microbiol. 2012, 50, 104-112. 